than 10 times during the previous year. The majority of those affected (97.4\%) reported that HAMP was triggered by prolonged MP use (mean time 49.7 +/- 36.7 minutes); $52.6 \%$ reported HAMP occurred every time they used a MP. The headache was mild in intensity, and dull or pressing in quality in 79\%, ipsilateral to the side of MP use (orbital or periorbital) in $84.2 \%$, associated with a burning sensation in $71.1 \%$ and dizziness in $39.5 \%$. Phonophobia was more prevalent among participants with HAMP compared to those without HAMP. The proportion of HAMP participants who suffered from migraine was $10.8 \%$, and among none-HAMP participants it was $8.4 \%$ (NS). (Chu MK, Song HG, Kim C, Lee BC. Clinical features of headache associated with mobile phone use: a crosssectional study in university students. BMC Neurology Nov 2011;11:115-122). (Respond: Dr MK Chu, Department of Neurology, Hallym University College of Medicine, Anyang, Korea. E-mail: 55brain@hallym.ac.kr).

COMMENT. The authors discuss the mechanism of HAMP and its possible relation to radiofrequency fields, psychological factors, temperature change, and noise. Only one participant in this cohort reported headache provocation by regular telephone use, and HAMP did not occur when using hands-free equipment. HAMP shows stereotyped clinical features.

\title{
METHYLENETETRAHYDROFOLATE REDUCTASE GENE VARIANT AND MIGRAINE
}

Researchers at McMaster University, Hamilton, ON, Canada performed a systematic review and meta-analysis of 15 studies of methylenetetrahydrofolate reductase gene (MTHFR C677T) and migraine in 447 patients selected from the Depression Case Control study (age 18 years or older). MTHFR C677T polymorphism was associated with migraine with aura (MA) $(\mathrm{p}=0.039)$, and the association remained significant after adjusting for age, sex and depression status. $\mathrm{T}$ allele homozygosity is significantly associated with MA and total migraine, but not migraine without aura. In studies of nonCaucasian population, the TT genotype was associated with total migraine, whereas in studies of Caucasians this variant was associated with MA only. (Samaan Z, Gaysina D, Cohen-Woods S, et al. Methylenetetrahydrofolate reductase gene variant (MTHFR C677T) and migraine: a case control study and meta-analysis. BMC Neurology Nov 2011;11:66-75). (Respond: Dr Z Samaan, Department of Psychiatry and Behavioral Neurosciences, McMaster University, Hamilton, ON, Canada. Email: samaanz@mcmaster.ca).

COMMENT. This study adds to the evidence for the role of MTHFR C677T gene variant in migraine with aura and shows a significant association between this genetic variant and MA in Caucasian population and total migraine in non-Caucasian population. A common polymorphism from the MTHFR gene, the C677T, is reported to be associated with both migraine and depression independently.

A population-based longitudinal community study of major depression and migraine in patients age $>12$ years found respondents with migraine were $60 \%$ more likely to develop major depressive episodes (MDE) compared with those without migraine. Similarly, respondents with MDE were 40\% more likely to develop migraine 
compared with those without MDE, but after adjustment for stress and childhood trauma, this association disappeared. Environmental factors such as childhood trauma and stress may shape the expression of this bidirectional relationship. (Modgill G et al. Headache Nov 15, 2011[Epub ahead of print]

\section{LANGUAGE-LEARNING DISORDERS}

\section{AUDITORY PROCESSING DISORDER AND CORTICAL MALFORMATION}

Researchers at Departments of Neurology and Speech, University of Campinas, Brazil assessed the auditory processing function in children with language-learning impairment in relation to malformation of cortical development. Thirty-two children (19 males), aged 8-15 years, were divided into three groups: Group I included 11 children with language-learning impairment and bilateral perisylvian polymicrogyria, a malformation shown to be associated with auditory processing disorders; Group II included 10 children with language-learning impairment and normal MRI; and Group III comprised 11 normal children. All patients had an IQ>80, only 1 had epilepsy, and 5 had pseudobulbar signs. Tests of auditory processing function, including the Random Gap Detection Test and Digits Dichotic Test, showed a statistically significant difference among the group. Groups I and II showed abnormalities in auditory processing when compared with the control group, and children in Group I were more affected than children in Group II. Perisylvian cortical malformation correlates with impairment of auditory processing function. This is expressed as difficulty in phonemic awareness, verbal comprehension, writing and reading, and processing of rapid auditory stimuli. (Boscariol M, Guimaraes CA, de Vasconellos Hage, SR, et al. Auditory processing disorder in patients with language-learning impairment and correlation with malformation of cortical development. Brain Dev Nov 2011;33:824-831). (Respond: MM Guerreiro. E-mail: mmg@,fcm.unicamp.br).

COMMENT. Cortical polymicrogyria malformation in the perisylvian regions may be associated with auditory processing dysfunction and language and learning disabilities, including dyslexia.

\section{LANGUAGE IMPAIRMENT, MOTOR DELAY AND ROLANDIC EPILEPSY}

Researchers at the Epilepsy Center and Department of Neurology, Maastricht University Medical Center, The Netherlands investigated a correlation between language, learning and locomotor impairments in a cohort of 48 children (6.5-13 years of age; 26 boys and 22 girls) with rolandic epilepsy referred to the Epilepsy Center between 2001 and 2009. EEG recordings and neuropsychological assessments were obtained within the same week. Parents completed a questionnaire on developmental milestones, attention, language, visuospatial skills, memory, reading, writing, and math. A learning efficacy quotient was calculated by dividing the educational level by months of education $\mathrm{x} 100$, 